\title{
Solvation at metal/water interfaces: An ab initio molecular dynamics benchmark of common computational approaches
}

Heenen, Hendrik H.; Gauthier, Joseph A.; Kristoffersen, Henrik H.; Ludwig, Thomas ; Chan, Karen

Published in:

Journal of Chemical Physics

Link to article, DOI:

$10.1063 / 1.5144912$

Publication date:

2020

Document Version

Peer reviewed version

Link back to DTU Orbit

Citation (APA):

Heenen, H. H., Gauthier, J. A., Kristoffersen, H. H., Ludwig, T., \& Chan, K. (2020). Solvation at metal/water interfaces: An ab initio molecular dynamics benchmark of common computational approaches. Journal of Chemical Physics, 152(14), [144703]. https://doi.org/10.1063/1.5144912

\section{General rights}

Copyright and moral rights for the publications made accessible in the public portal are retained by the authors and/or other copyright owners and it is a condition of accessing publications that users recognise and abide by the legal requirements associated with these rights.

- Users may download and print one copy of any publication from the public portal for the purpose of private study or research.

- You may not further distribute the material or use it for any profit-making activity or commercial gain

- You may freely distribute the URL identifying the publication in the public portal 
Solvation at metal/water interfaces: An ab initio molecular dynamics benchmark of common computational approaches

Hendrik H. Heenen, ${ }^{1}$ Joseph A. Gauthier, ${ }^{2,3}$ Henrik H. Kristofferson, ${ }^{1}$ Thomas

Ludwig, ${ }^{2,3}$ and Karen Chan ${ }^{1, \text { a) }}$

1) Department of Physics, Technical University of Denmark, DK-2800, Kgs. Lyngby,

Denmark

2) SUNCAT Center for Interface Science and Catalysis,

Department of Chemical Engineering, Stanford University, Stanford, California 94305, United States

3) SUNCAT Center for Interface Science and Catalysis,

SLAC National Accelerator Laboratory, 2575 Sand Hill Road, Menlo Park, California 94025, United States

(Dated: 13 March 2020) 
Rationalizing the influence of the solvent on electrochemical reaction energetics is a central challenge in our understanding of electrochemical interfaces. To date, it is unclear how well existing methods predict solvation energies at solid/liquid interfaces since they cannot be assessed experimentally. Ab initio molecular dynamics (AIMD) simulations present a physically highly accurate, but also a very costly approach. In this work, we employ extensive AIMD simulations to benchmark solvation at charge-neutral metal/water interfaces against commonly applied continuum solvent models. We consider a variety of adsorbates including ${ }^{*} \mathrm{CO},{ }^{*} \mathrm{CHO},{ }^{*} \mathrm{COH},{ }^{*} \mathrm{OCCHO}$, $* \mathrm{OH}$, and ${ }^{*} \mathrm{OOH}$ on $\mathrm{Cu}, \mathrm{Au}$, and $\mathrm{Pt}$ facets solvated by water. The surfaces and adsorbates considered are relevant, among other reactions, to electrochemical $\mathrm{CO}_{2}$ reduction and the oxygen redox reactions. We determine directional hydrogen bonds and steric water competition to be critical for a correct description of solvation at the metal/water interfaces. As a consequence, we find that the most frequently applied continuum solvation methods, which do not yet capture these properties, do not presently provide more accurate energetics over simulations in vacuum. We find most of the computed benchmark solvation energies to linearly scale with hydrogen bonding or competitive water adsorption, which strongly differ across surfaces. Thus, we determine solvation energies of adsorbates to be non-transferable between metal surfaces in contrast to standard practice.

a)Electronic mail: kchan@fysik.dtu.dk 


\section{INTRODUCTION}

Modeling the electrochemical interface via atomistic simulations is a central challenge in computational electrochemistry. In a comprehensive atomistic treatment, both a description of the electrified double layer under the influence of an external potential and the interaction of reaction intermediates with the solvent (e.g. water) are required ${ }^{1}$. In density functional theory (DFT) calculations, recent approaches to represent the solid/liquid interface are solid/vacuum interfaces with a posteriori corrections for solvation ${ }^{2,3}$, "implicit" continuum solvation methods ${ }^{4,5}$, static ice-like water bilayers ${ }^{6,7}$, and ab initio molecular dynamics (AIMD) simulations ${ }^{8-10}$.

Implicit solvation methods employ a polarizable dielectric continuum to represent the solvent. The continuum forms a cavity around a solute by varying the dielectric constant $\epsilon$ smoothly from the bulk solvent value (i.e. for water $\epsilon \approx 80$ ) to the vacuum permittivity where the quantum mechanical electron density is located. The parameterized interaction of the dielectric mimics an ensemble representation of the solvent but usually does not include directional interactions with the solvent (e.g. hydrogen bonding), unless specifically accounted for (e.g. in the RISM approach ${ }^{11}$ or CANDLE ${ }^{12}$ solvent model). In combination with an electrolyte model, e.g. a modified Poisson-Boltzmann model, a tunable interfacial potential can be introduced ${ }^{13-15}$. In contrast, explicit, static ice-like bilayer models include directional water interactions but lack an ensemble effect. They therefore suffer from a sampling problem originating in a plethora of shallow local minima corresponding to different water structures. If not sampled consistently, the computed solvation energies may not be reliable ${ }^{16}$. Through the combination of static water bilayers with implicit/electrolyte models, a potential control can be introduced ${ }^{17-21}$. AIMD represent a highly accurate, but also a very computationally costly approach. By simulating the time propagation of a water ensemble, temperature effects of the directional water interactions are accounted for. Currently, the control of the potential during such simulations remains an open challenge, with pioneering work only recently emerging ${ }^{22,23}$.

From a practical point of view, the application of implicit solvent models is highly attractive. The continuum modelling approach not only yields minimal computational cost through the efficient representation of the solvent, but also through the elimination of the sampling problem. Combined with the potential control enabled by continuum electrolyte 
models, these methods present the ideal tool to investigate electrochemical problems. It is, however, unclear how well these methods capture solvation at the solid/liquid interface. This

is due to the fact that any parameterization is based on solvation references in bulk solvent ${ }^{4,12}$ and directional and steric interactions with explicit water molecules are absent ${ }^{1,15}$, at least for the most frequently used models. Similarly, any improvement in accuracy via the inclusion of static water molecules is in no way confirmed. Thus, the accuracy or shortcomings of the application of implicit solvation methods for solid/liquid interfaces remain enigmatic. Without an experimental benchmark for solvation energies at solid/liquid interfaces ${ }^{1}$, AIMD simulations present a reliable way to quantify solvation energies at solid/liquid interfaces.

In this work, we perform a benchmark for solvation effects at uncharged metal/water interfaces via AIMD simulations. We focus on metal surfaces, since these are well-behaved at the GGA-DFT level. Our test set comprises of a variety of electrocatalytically relevant adsorbates and metal surfaces with a wide range of binding strengths: we investigate ${ }^{*} \mathrm{CO}$, *OH on $\mathrm{Cu}(111), \mathrm{Au}(111)$, and $\mathrm{Pt}(111),{ }^{*} \mathrm{OOH}$ on $\mathrm{Au}(111)$ and $\mathrm{Pt}(111)$ as well as ${ }^{*} \mathrm{CO}$, ${ }^{*} \mathrm{CHO},{ }^{*} \mathrm{COH},{ }^{*} \mathrm{OCCHO}$, and ${ }^{*} \mathrm{OH}$ on $\mathrm{Cu}(211)$. We quantify and rationalize the solvation energies obtained. In that, directional hydrogen bonds and steric competitive water adsorption $^{24}$ appear critical and linearly correlate with the solvation energies of adsorbates with specific functional groups. We find that solvation energies of individual adsorbates vary strongly across metals and facets, which impedes their transferability for a posteriori corrections. The solvation energies determined from frequently applied continuum solvent methods strongly deviate from those determined from AIMD due to the missing directional and steric solvation effects. Specifically for our diverse test-set, the associated mean absolute errors are equivalent to those obtained from pure solid/vacuum calculations. On the one hand, these results put to question the accuracy of recent popular implicit solvent models for solvation energies of adsorbates. On the other hand, they provide a reference for future developments of these effective/parameterizable models. 


\section{METHODS}

\section{A. Computational Details}

Density functional theory (DFT) calculations are performed with the Vienna Ab Initio Simulation Package (VASP) ${ }^{25,26}$ employing the projector-augmented wave (PAW) $\operatorname{method}^{27}$ in combination with a plane wave basis set in periodic boundary conditions. The $\mathrm{RPBE}^{28}$ exchange-correlation functional is used and dispersion effects are included via the semiempirical zero damping D3 dispersion correction scheme of Grimme ${ }^{29,30}$. This "RPBE-D3" setup has been reported to accurately simulate liquid water properties on metal surfaces ${ }^{31-33}$. A Gaussian smearing with a width of $0.1 \mathrm{eV}$ and plane wave cutoff energies of $400 \mathrm{eV}$ are chosen. Electronic structure calculations are converged until a total energy difference of $10^{-4} \mathrm{eV}$ whereby a quantitatively consistent performance against a more accurate convergence criterion of $10^{-6} \mathrm{eV}$ is ensured (via the energy conservation and relative energetics in AIMD runs). For geometry optimizations, all atomic structures are relaxed until residual forces are less than $0.05 \mathrm{eV} / \AA$. In the AIMD simulations the motion of atoms is treated classically using a 1 fs timestep within the NVT ensemble by employing a Nose thermostat at $300 \mathrm{~K}^{34-36}$.

To simulate metal surfaces, we use slab models with a $3 \times 1$ and $3 \times 4$ supercell for the fcc(211) and fcc(111) facets, respectively. A $4 \times 4 \times 1$ and $4 \times 3 \times 1 \Gamma$-point centered MonkhorstPack k-point grid is applied to sample the Brillouin zone ${ }^{37}$. The lattice constants obtained for the employed RPBE-D3 scheme based on a $1 \times 1 \times 1$ bulk unit cell and a k-point den-

sity consistent $12 \times 12 \times 12 \mathrm{k}$-point grid are $3.560 \AA$ for $\mathrm{Cu}, 4.167 \AA$ for $\mathrm{Au}$, and $3.934 \AA$ for Pt. AIMD simulations are conducted using (non-symmetric) slabs of three layers fixing the positions of the two bottom layers and including a dipole correction ${ }^{38}$. Geometry optimizations are conducted on symmetric slabs of four layers with the two central layers fixed. The symmetric cells are used to ensure a reliable determination of the workfunction if continuum solvation is applied ${ }^{18}$. To avoid interactions across periodic images, a vacuum spacing of 10 $\AA$ on each side of the slab is included. For convenient handling of atomic structures, the Atomic Simulation Environment (ASE) package is used ${ }^{39}$.

Solvation at the metal surface is modeled via (1) AIMD simulations of explicit water molecules (see below Sec. II B) and (2) an electron density dependent continuum solvent 
model $^{40}$ as implemented in VaspSol ${ }^{5}$ combined with geometry optimizations. Solvation-free reference calculations are conducted for (1) via AIMD in absence of explicit water molecules and for (2) via geometry optimization in absence of continuum solvation. To generalize our findings with respect to the implicit solvation method, we compare the VaspSol continuum solvation and the SCCS model in Quantum ESPRESSO / Environ ${ }^{4,41}$. We find them to yield near-identical solvation energies, c.f. Supporting Information (SI) Sec. III. All simulations of (adsorbates at) the metal/water interface do not include a net charge on the metal slab and thus correspond to the model systems at the potential of zero charge (PZC).

\section{B. AIMD Statistics and Evaluation}

To ensure high accuracy of the AIMD simulations, a physically sound representation of the solid/liquid interface through a sufficient number of water molecules in the atomic model and well converged statistics are required. The metal/water interface is modeled by roughly three "layers" of explicit water molecules, resulting in 15 and $24 \mathrm{H}_{2} \mathrm{O}$ for the $3 \times 1$ fcc $(211)$ and $3 \times 4$ fcc(111) facet, respectively. For each surface-adsorbate combination, four individual AIMD runs are conducted starting from different water structures. The respective initial water structures are local minima which deviate by up to $0.5 \mathrm{eV}$ in relative energy. The different starting points allow for a distinct randomization among the varying trajectories. This setup avoids an entrapment in one local minimum of the water structure which may potentially occur if only a single trajectory is investigated. Each MD trajectory is run for a duration of $>30 \mathrm{ps}$, whereby data sampling is commenced after an equilibration period of 5 ps yielding in total at least 100 ps per surface-adsorbate combination. We ensured that the individual trajectories are converged showing a drift in total energy of $<0.03 \mathrm{eV} / \mathrm{ps}$ after equilibration (c.f. SI Sec. I). We determine an uncertainty for all averaged quantities from the standard deviation $\sigma$ between the $n(=4)$ individual MD runs as $\frac{\sigma}{\sqrt{n}}$.

Within the 3 water layers on the $\mathrm{Cu}$ and Pt surfaces, we find a "bulk" water segment with a thickness of $5-7 \AA$, where we have defined "bulk" to correspond to regions with $\geq 0.95$ of the experimental water density in agreement with previous work ${ }^{32,33,42}$ (c.f. Fig. 4 and 5). We also find that in these regions the $\mathrm{O}$ and $\mathrm{H}$ density profiles are relatively flat and featureless. The corresponding region on $\mathrm{Au}(111)$ appears slightly less dense with $\geq 0.85$ of the experimental water density. As demonstrated in the SI Sec. VII, the decreased "bulk" density arises 
from the size of the water film considered in the model system. When including more water molecules, the "bulk" region converges to 0.99 of the experimental water density. The thicker water film slightly increases the amount of water molecules adsorbed on $\mathrm{Au}(111)$ and destabilizes the solvation energy of $* \mathrm{OH}$ by $\approx 0.1 \mathrm{eV}$. We expect this minor size effect only for $\mathrm{Au}(111)$, since this is the only metal surface showing a reduced density in its "bulk" region in our 3 water layer setup. For Pt(111), we do not find the "bulk" density or interface properties altered when going beyond the 3 water layers, demonstrating convergence with 3 water layers (c.f. SI Sec. VII). These results are in line with the previously reported observation that the characteristics of the metal/water interface have been found rather insensitive to the absolute water "bulk" thickness and extended system size ${ }^{42}$. Thus, accurate solvation effects can be expected from our employed setup. Further, our Pt(111)/water interface with small bulk water thickness yields a work function of $4.86 \pm 0.32 \mathrm{eV}$ which compares well to the value of $4.96 \pm 0.23 \mathrm{eV}$ found for a considerably thicker (6 water layer/Pt(111)) interface using the same DFT settings ${ }^{32,33}$. We find that the work function of $\mathrm{Au}(111)$ shows the same convergence behaviour (c.f. SI Sec. VII). Reference AIMD simulations are conducted for all surface-adsorbate combinations without solvent and for gas phase molecules. We ran these reference calculations once, since convergence is rapidly achieved and the variation between runs is negligible.

We compare internal adsorption energies $\Delta E_{\mathrm{ad}}\left({ }^{*} A \mid M e\right)$ of various adsorbates ${ }^{*} A$ on the metal surfaces $M e$ as calculated via the different approaches to solvation. These relative energies are evaluated by

$$
\Delta E_{\text {ad }}\left({ }^{*} A \mid M e\right)=E\left({ }^{*} A+M e_{\text {slab }}\right)-E\left(M e_{\text {slab }}\right)-E\left(A_{\mathrm{g}}\right)
$$

where $E\left({ }^{*} A+M e_{\text {slab }}\right), E\left(M e_{\text {slab }}\right), E\left(A_{\mathrm{g}}\right)$ correspond to the energy of the adsorbate on the metal slab, the pristine metal slab and the adsorbate gas phase reference, respectively. The states * $A+M e_{\text {slab }}$ and $M e_{\text {slab }}$ can thereby correspond to systems with solvation (based on AIMD or continuum solvent) or without solvation (solid/gas interface). All adsorbates are referenced to the gas phase molecules $\mathrm{H}_{2}, \mathrm{H}_{2} \mathrm{O}$ and $\mathrm{CO}$. It follows for the gas phase reference energy $E\left(A_{\mathrm{g}}\right)$

$$
E\left(A_{\mathrm{g}}\right)=\frac{n_{\mathrm{H}}}{2} E\left(\mathrm{H}_{2}\right)+n_{\mathrm{O}}\left(E\left(\mathrm{H}_{2} \mathrm{O}\right)-E\left(\mathrm{H}_{2}\right)\right)+n_{\mathrm{C}}\left(E(\mathrm{CO})-E\left(\mathrm{H}_{2} \mathrm{O}\right)+E\left(\mathrm{H}_{2}\right)\right)
$$

where $n_{\mathrm{H}}, n_{\mathrm{O}}$, and $n_{\mathrm{C}}$ are the number of $\mathrm{H}, \mathrm{O}$, and $\mathrm{C}$ species in the adsorbate, respectively. When handling AIMD energetics, internal energies correspond to the sum of the 
time averaged potential and kinetic energy. Also, we add $\frac{3}{2} \mathrm{k}_{\mathrm{B}} T$ to the energy of each stoichiometric gas phase reference molecule, since the center of mass motions are not included in the gas-phase MD simulations ${ }^{43,44}$. It should be noted that solvation energies obtained with continuum solvation include the free energy contribution (containing the entropy cost) of forming a solvation shell around the solute molecule. In contrast, solvation energies obtained from AIMD do not include entropy effects. Fortunately, inserting the adsorbates on the metal surfaces only lead to a minor increase in the solvent accessible surface $\operatorname{areas}^{45}$, which correspond to free energy contributions of $<0.06 \mathrm{eV}$ as we demonstrate in the SI Sec. VI. A direct comparison of adsorption and solvation energies between the different methods is therefore justified.

We carefully monitor the microscopic events occurring during the AIMD trajectories to capture the mobility and integrity of the adsorbates, hydrogen bonding, and $\mathrm{H}_{2} \mathrm{O}$ adsorption. The diffusion of adsorbates and water molecules is tracked via the mean squared displacement (MSD). The increased mobility at the water/vacuum interface is omitted from the diffusion analysis through exclusion of water molecules which reside within this region for more than $5 \%$ of a trajectory. The water/vacuum region is defined within $4 \AA$ from the outer most $\mathrm{H}_{2} \mathrm{O}$ at a given snapshot. To remove noise, the MSD is time averaged from overlapping subsets of atom displacement and diffusion coefficients are evaluated following the Einstein formulation ${ }^{46,47}$.

The instantaneous adsorption site of each adsorbate is tracked by the distance to the individual surface sites (c.f. Fig. 1) which are determined using the package CatKit ${ }^{48}$. For each snapshot all distances $d$ to the surface sites are weighted by a Gaussian $f_{g}=\exp \left(-d^{2} / 2 \sigma^{2}\right)$ and normalized. The standard deviation $\sigma$ for the distance weighting Gaussian is chosen to drop to virtually $\approx$ zero $(0.1 \%)$ within the shortest distance between two surface sites (c.f. Fig. 1). This approach gives a quantitative measure of the fractional site occupation which allows us to (1) obtain the share of site occupation over the course of a trajectory and (2) partition the AIMD energies to single sites. For the latter, an individual site is counted as fully occupied if the normalized Gaussian weight is $>50 \%$ (compare Fig. 1). Dissociation and desorption events, as well as other chemical reactions (c.f. Sec. III A) are tracked and the corresponding parts of the trajectories are omitted from the evaluation of the energetics. Hydrogen bonds are evaluated following the criterion of O-H-O angles $>140{ }^{\circ}$ between an $\mathrm{O}$ atom of an adsorbate and of a water molecule which is coordinating the former within 

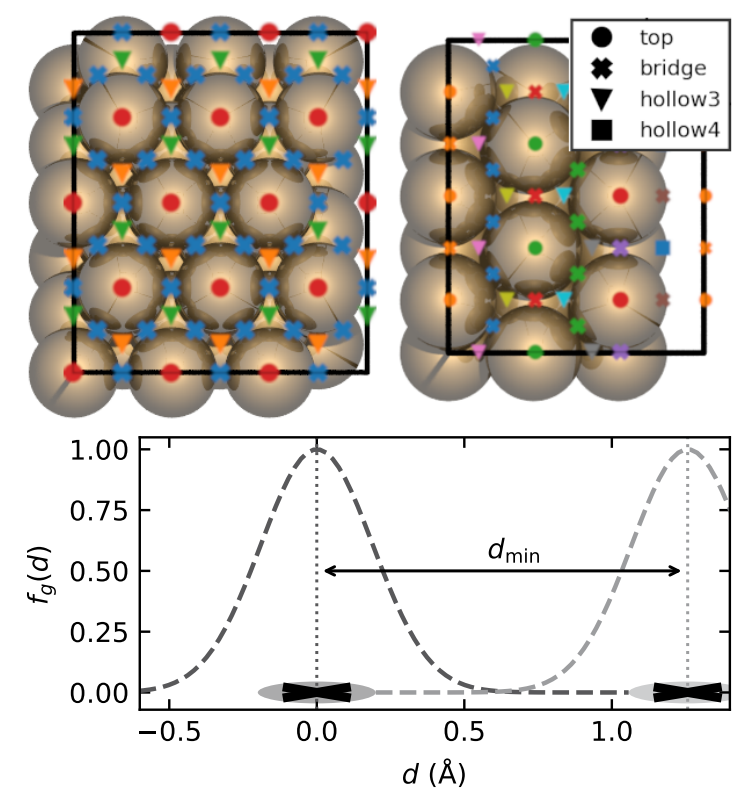

FIG. 1. Surface sites for the $3 \times 4-(111)$ (top left) and $3 \times 1-(211)$ (top right) slab models used for the site-partitioning of the AIMD trajectories. Symmetrically equivalent sites are shown in the same color. (bottom) Schematic of Gaussian weights on surface sites with Gaussian weighting function $\left(f_{g}\right)$ against the distance $d$. The weighting function overlap is depicted for two sites separated by the minimum distance $d_{\text {min }}$.

a distance of $3.5 \AA^{49,50}$. Water adsorption is tracked by monitoring the distance of water molecules to the surface atoms. A water molecule is considered adsorbed if it is closer than $2.55 \AA$ to the position of a surface atom. We determined this criterion via radial distribution functions as discussed in the SI Sec. II. When evaluating the frequency of chemical reactions or water adsorption, recrossing events are omitted if the lag time between them is $<150$ fs. This lag time is chosen to optimize the distinctness of the ad/desorption process of water molecules on the metal surface. 


\section{RESULTS AND DISCUSSION}

\section{A. The thermalized metal/water interface}

\section{Dynamic vs. static adsorbates}

A continuum dielectric and AIMD simulation with explicit water molecules give, respectively, a static $0 \mathrm{~K}$ picture and a thermalized ensemble average. In the former case, the model system is an adsorbate resting on a single site, while in the latter, the adsorbate is subject to surface diffusion and interaction with explicit water molecules. As described in Sec. IIB, the diffusion of adsorbates and water is monitored via the MSD, which allows us to determine diffusion coefficients. Nearly all investigated adsorbates on all surfaces show a high degree of mobility, as shown in Fig. 2. This mobility is consistent with the low diffusion barriers on metal surfaces ${ }^{51}$. Additionally, the mobility scales approximately with the binding strength, as visible for ${ }^{*} \mathrm{CO}$ on the different surfaces with the trend $\mathrm{Au}(111) \geq \mathrm{Cu}(111)>\mathrm{Cu}(211)>\operatorname{Pt}(111)$; the adsorption energies $\Delta E_{\text {ad }}$ are $-0.26,-0.77$, -0.69 , and $-2.07 \mathrm{eV}$, respectively (c.f. Fig. 8 and SI Sec. III). Water is very mobile in all simulations, indicating a high degree of averaging for the solvent interactions around the various adsorbates. The numerical diffusion coefficients for water are in most cases close to the experimental value of $\approx 0.23 \AA^{2} / \mathrm{ps}^{52}$. The maximum deviation of the water diffusion coefficients is a factor of only 2 and may be functional related (as known for revPBE) ${ }^{53}$.

Besides a high surface mobility of adsorbates, chemical reactions of the adsorbates occur in some AIMD simulations, as schematically depicted in Fig. 3. ${ }^{*} \mathrm{OH}$ adsorbates desorb as $\mathrm{H}_{2} \mathrm{O}$ after their protonation by co-adsorbed water molecules which simultaneously form new $* \mathrm{OH}$ adsorbates. As shown in Tab. I, the frequency of $* \mathrm{OH}$ to $\mathrm{H}_{2} \mathrm{O}$ conversion strongly differs for each surface. Further, ${ }^{*} \mathrm{CO}$ reversibly desorbs from $\mathrm{Au}(111)$ due to its weak binding. The desorbed state of ${ }^{*} \mathrm{CO}$ makes up $44 \%$ of the sampled run time. Dissociations of $\mathrm{H}$ from ${ }^{*} \mathrm{CHO}$ and ${ }^{*} \mathrm{COH}$ to form the more stable ${ }^{*} \mathrm{CO}$ occur irreversibly within the timescales of

our simulations. ${ }^{*} \mathrm{CHO}$ dissociates to ${ }^{*} \mathrm{H}$ and ${ }^{*} \mathrm{CO}$ in two of four trajectories, with the dissociated state making up $32 \%$ of the sampled run time. $\mathrm{COH}^{*}$ is deprotonated by water to form ${ }^{*} \mathrm{CO}$ and $\mathrm{H}_{3} \mathrm{O}^{+}$, simultaneously charging the $\mathrm{Cu}(211)$ metal slab. This event occurs within all trajectories, with the deprotonated state making up $47 \%$ of the sampled run time. Lastly, ${ }^{*} \mathrm{OOH}$ on $\mathrm{Pt}(111)$ deprotonates or dissociates irreversibly in our AIMD timescales 

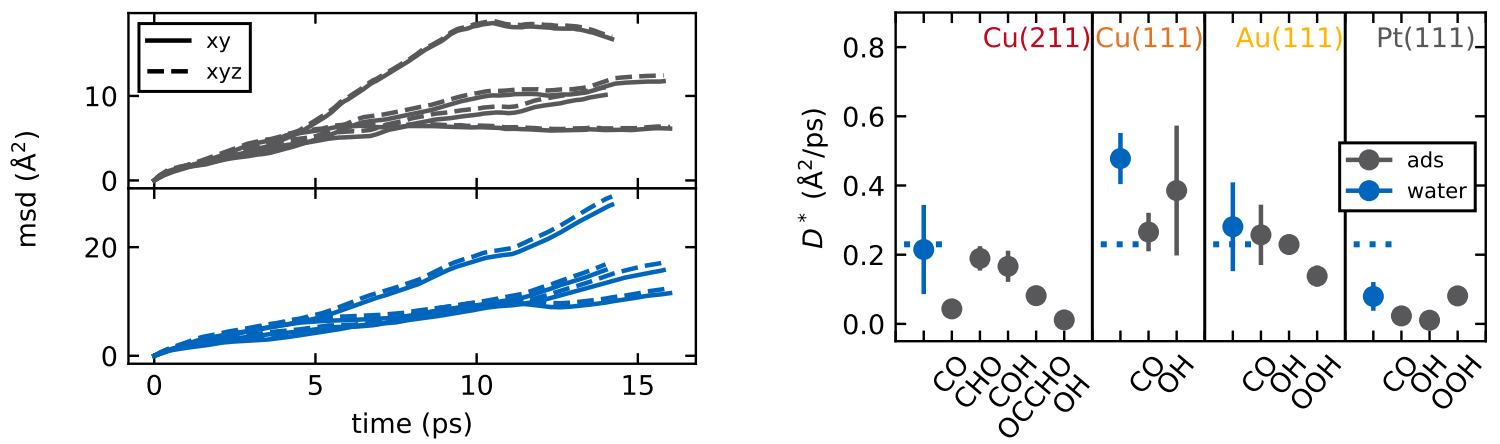

FIG. 2. (left) Time averaged mean squared displacement (MSD) of the 4 AIMD trajectories for the example of ${ }^{*} \mathrm{CHO}$ on $\mathrm{Cu}(211)$. The $\mathrm{MSD}$ of ${ }^{*} \mathrm{CHO}$ is shown above (grey lines) and of water below (blue lines). (right) Averaged diffusion coefficients of all water and all investigated adsorbates on $\mathrm{Cu}(211), \mathrm{Cu}(111), \mathrm{Au}(111)$, and $\mathrm{Pt}(111)$. The diffusion coefficients of water were obtained from an average over all simulations, with and without adsorbates. The horizontal dotted blue lines indicate the experimental diffusion coefficient of water. ${ }^{52}$

to form ${ }^{*} \mathrm{O} 2$ and ${ }^{*} \mathrm{O}+{ }^{*} \mathrm{OH}$, respectively. Each event occurs in one trajectory, comprising up $15 \%$ and $5 \%$ of the sampled run time. On a side note, ${ }^{*} \mathrm{OOH}$ dissociation within the first $100 \mathrm{fs}$ was also found on all $\mathrm{Cu}$ surfaces, which is why ${ }^{*} \mathrm{OOH}$ on $\mathrm{Cu}$ was omitted in this study. The parts of the trajectories where ${ }^{*} \mathrm{CHO},{ }^{*} \mathrm{COH}$, and ${ }^{*} \mathrm{OOH}$ are dissociated or ${ }^{*} \mathrm{CO}$ is desorbed are excluded from the calculation of adsorption energies and other properties.

TABLE I. Statistics of ${ }^{*} \mathrm{OH}$ to $\mathrm{H}_{2} \mathrm{O}$ conversion. Given are the frequency of $* \mathrm{OH}$ conversion $f$ and the number of observed events $n_{\text {events }}$ during combined sampling time of $\approx 100 \mathrm{ps}$ for each surface. The data is corrected for recrossing events as described in Sec. II B.

\begin{tabular}{c|cc}
\hline & $f\left(10^{4} / \mathrm{s}\right)$ & $n_{\text {events }}$ \\
\hline $\mathrm{Cu}(211)$ & 5.0 & 5 \\
$\mathrm{Cu}(111)$ & 40.3 & 41 \\
$\mathrm{Au}(111)$ & 13.9 & 14 \\
$\mathrm{Pt}(111)$ & 102.3 & 102 \\
\hline
\end{tabular}




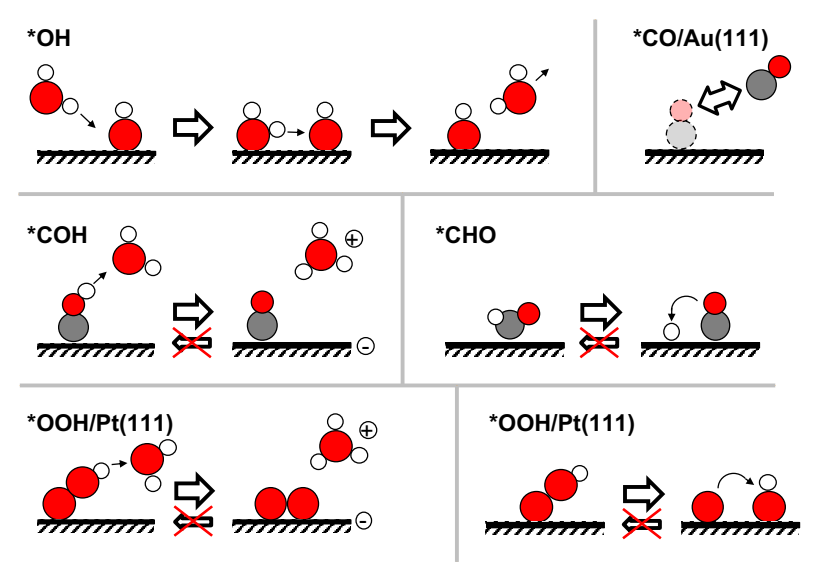

FIG. 3. Schematic of possible reactions occurring during the AIMD trajectories: (top left) $* \mathrm{OH}$ to $\mathrm{H}_{2} \mathrm{O}$ conversion, (top right) ${ }^{*} \mathrm{CO}$ desorption on $\mathrm{Au}(111)$, (center left) ${ }^{*} \mathrm{COH}$ deprotonation, (center right) $* \mathrm{CHO}$ dissociation, (bottom left) $* \mathrm{OOH}$ deprotonation on $\mathrm{Pt}(111)$, (bottom right) *OOH dissociation on $\mathrm{Pt}(111)$.

\section{Water-metal interaction}

We assess the interaction of water with the metal surfaces through the average atomic density profiles normal to the surface as well as water adsorption. The former are shown in Fig. 4 and 5. All surfaces show a maximum density at the immediate solid/liquid interface. The features of this high-density region mark the differences between the clean metal/water interfaces considered, as shown in the top panels of Fig. 4 and 5. Pt(111) shows a pronounced double O-peak, indicative of a bilayer structure ${ }^{9,32,44,54}$. Consistent with a previous report ${ }^{42}$, this feature appears to a lesser degree on $\mathrm{Cu}(111)$. On $\mathrm{Au}(111)$, only a single broad peak is found, which indicates the absence of any bilayer water structure. In accordance with the reduced degree of structuring, the mean position of the first water layer is farther from the $\mathrm{Au}(111)$ surface at $2.5 \AA$ in comparison to $2.0 \AA$ for $\mathrm{Cu}(111)$ and for $\operatorname{Pt}(111)$. The stepped $\mathrm{Cu}(211)$ does not show a double peak feature in the presented densities along the surface normal. As shown via radial distribution functions in the SI Fig. S2, a bilayer structure is however present at the step-edge of $\mathrm{Cu}(211)$. In fact, a very strong interaction with water can be detected with the first "layer" located at $1.9 \AA$ from the step edge.

The water-metal interaction is also indicated through water adsorption, which is evaluated as described in Sec. II B. The adsorbing water molecules thereby approach the metal surface for short periods of time. Water adsorbs on the top sites of the (111) terraces and on the top 

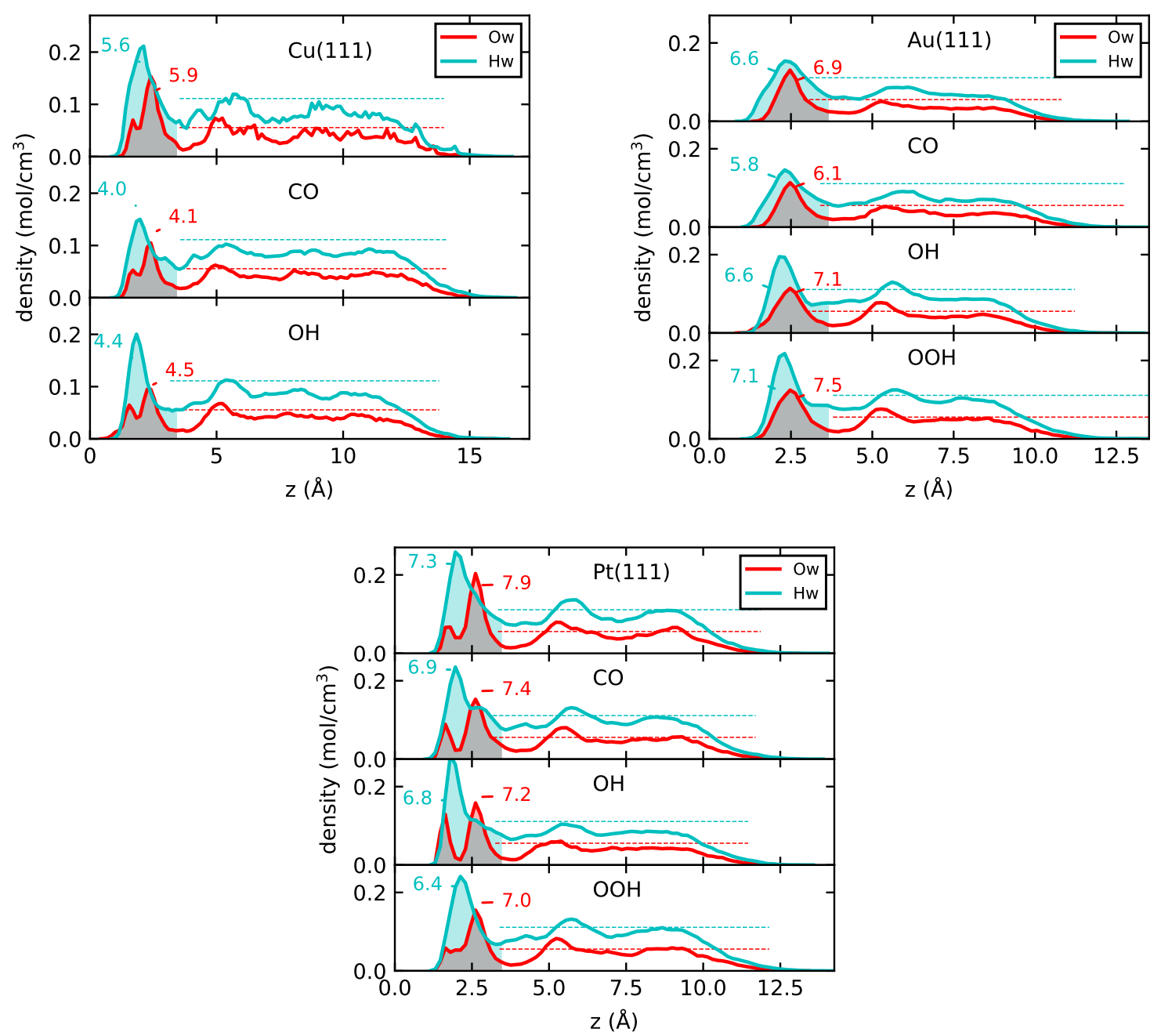

FIG. 4. Average atomic densities of $\mathrm{Cu}(111)$ (top left), $\mathrm{Au}(111)$ (top right), and $\mathrm{Pt}(111)$ (bottom) along the surface normal $z$. The densities for the clean surfaces and in presence of ${ }^{*} \mathrm{CO},{ }^{*} \mathrm{OH}$, and * $\mathrm{OOH}$ are depicted as labelled. The atomic density of $\mathrm{O}\left(\mathrm{O}_{\mathrm{w}}\right)$ and $\mathrm{H}\left(\mathrm{H}_{\mathrm{w}}\right)$ from water are shown in red and cyan, respectively. The experimental atomic densities of bulk water are depicted by the dotted lines. The shaded area corresponds to the integrated area of the first water layer with the numeric values included, normalized to $\mathrm{H}_{2} \mathrm{O}$ molecules per unit cell.

sites at step edges on $\mathrm{Cu}(211)$ as detailed in the SI Sec. IV. The amount of water adsorption is depicted in Fig. 6. For the clean surfaces, there is a trend in the average number of $\mathrm{H}_{2} \mathrm{O}$ molecules adsorbed $\left(\left\langle n_{\text {ads }}^{\mathrm{H}_{2} \mathrm{O}}\right\rangle\right)$ following $\mathrm{Pt}(111)>\mathrm{Cu}(211)>\mathrm{Cu}(111)>>\mathrm{Au}(111)$. Pt binds individual water molecules for periods of around 1 ps whereas the exchange on $\mathrm{Cu}$ is much more rapid with $\mathrm{H}_{2} \mathrm{O}$ residence times of $<500 \mathrm{fs}$. $\mathrm{Au}(111)$ shows a very weak binding of 


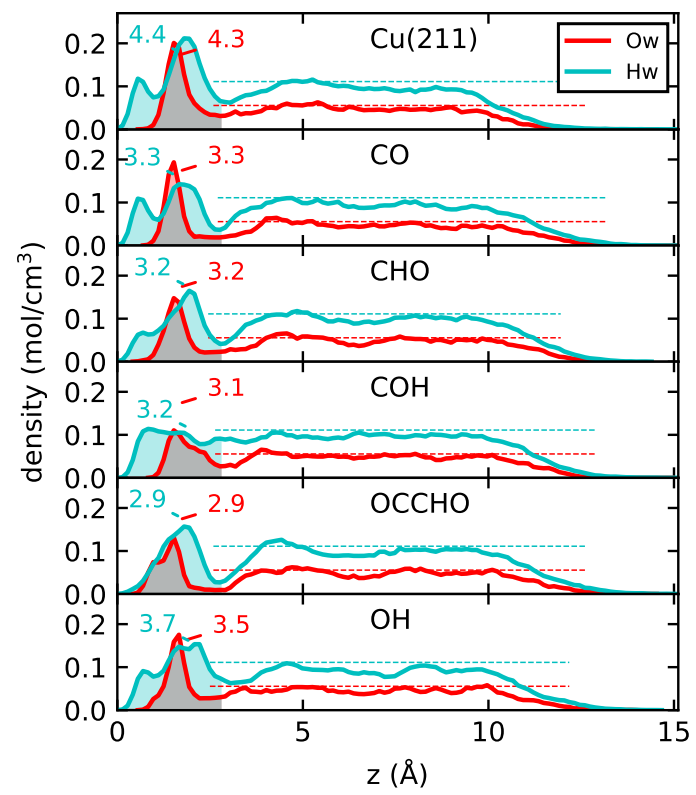

FIG. 5. Average atomic densities of $\mathrm{Cu}(211)$ along the surface normal $z$, on a clean surface and in presence of ${ }^{*} \mathrm{CO},{ }^{*} \mathrm{CHO},{ }^{*} \mathrm{COH}$ and ${ }^{*} \mathrm{OCCHO},{ }^{*} \mathrm{OH}$ (as labelled). The atomic density of $\mathrm{O}\left(\mathrm{O}_{\mathrm{w}}\right)$ and $\mathrm{H}\left(\mathrm{H}_{\mathrm{w}}\right)$ from water are shown in red and cyan, respectively. The experimental atomic densities of bulk water are depicted by the dotted lines. The shaded area corresponds to the integral of the first water layer with the numeric values included, normalized to $\mathrm{H}_{2} \mathrm{O}$ molecules per unit cell.

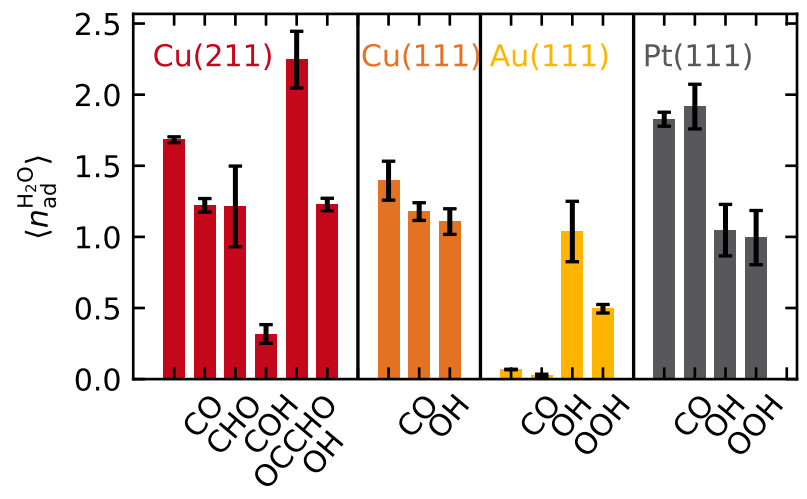

FIG. 6. Average number of water molecules adsorbed $\left(\left\langle n_{\mathrm{ad}}^{\mathrm{H}_{2} \mathrm{O}}\right\rangle\right)$ in the computational cell for $\mathrm{Cu}(211), \mathrm{Cu}(111), \mathrm{Au}(111)$, and $\mathrm{Pt}(111)$ with and without (no label) the presence of adsorbates.

water to the bare metal surface. The presented values for $\mathrm{Au}(111)$ are, however, slightly underestimated, due to size effects of the employed water thickness (c.f. SI Sec. VII).

Both the density profiles and water adsorption show a trend in the overall hydrophilicity 
of the metal surfaces in the order $\mathrm{Pt}>\mathrm{Cu}>\mathrm{Au}$. Such a trend cannot be captured in the effective, averaged nature of implicit solvation methods. There is of course an effect based on the placement of the dielectric continuum against the electron density in self-consistent models ${ }^{4,14}$, but its qualitative implication is hardly assessed. As we discuss below in Sec. III C, water adsorption as a consequence of this hydrophilicity has a significant impact on adsorbate solvation.

\section{Water-adsorbate interaction}

The interaction of water with a polar adsorbate occurs predominantly through hydrogen bonding. As described in Sec. IIB, we use a structural criterion to identify hydrogen bonds in an AIMD snapshot. Of the identified hydrogen bonds to adsorbates, we compute time averages $\left(\left\langle n_{\text {Hbond }}\right\rangle\right)$ which are presented in Tab. II. The affinity for hydrogen bonding shows a stronger correlation with the identity of the adsorbate than with the identity of the surface. ${ }^{*} \mathrm{CO}$ has the weakest affinity, with $<0.5$ hydrogen bonds on average $\left(\left\langle n_{\text {Hbond }}\right\rangle\right)$, ${ }^{*} \mathrm{CHO}$ and ${ }^{*} \mathrm{COH}$ show an intermediate affinity with $\left\langle n_{\mathrm{Hbond}}\right\rangle=1.0-2.0$ whereas $* \mathrm{OH},{ }^{*} \mathrm{OOH}$ and $*$ OCCHO show the strongest affinity, with $\approx 2.0-3.5\left\langle n_{\text {Hbond }}\right\rangle$. A particularly high variation in the hydrogen bonding amongst different trajectories is seen for *OCCHO. This variation is due to configurational changes around the O-C-C-O dihedral angle. While a dihedral angle around $0-90^{\circ}$ is usually present, a dihedral angle of $\approx 180^{\circ}$ is sometimes found. The latter configuration leads to reduced hydrogen bonding since the oxygen is sterically less accessible.

The hydrogen bonding affinity correlates with water density at the interface, as indicated by the density integral of the first water layer (c.f. Fig. 4 and 5). Adsorbates with a weak affinity repel water and thereby reduce the density in the first water layer through the formed cavity. In comparison, adsorbates with strong affinities for hydrogen bonds integrate into the water structure and maintain the pristine water density on the surface. On the (111) terraces, ${ }^{*} \mathrm{OH}$ and ${ }^{*} \mathrm{OOH}$ have a very similar water density to that of the pristine surface. Due to the reduced space on the (211) step-edge leading to $\mathrm{O}-\mathrm{O}$ repulsion, ${ }^{*} \mathrm{OH},{ }^{*} \mathrm{COH}$, and * OCCHO show this effect only in the H-density of the water. We find that the hydrogen bonding of the adsorbates "pulls" the water to the surface, which is especially apparent for $* \mathrm{OH}$ on $\mathrm{Au}(111)$. As shown in Fig. 6, the ${ }^{*} \mathrm{OH}$ and $* \mathrm{OOH}$ adsorbate even induce 
TABLE II. Hydrogen bond statistics for the different adsorbates on the investigated surfaces. Displayed are the average number of hydrogen bonds $\left(\left\langle n_{\mathrm{Hbond}}\right\rangle\right)$, the deviation in the average value between different AIMD runs $\sigma_{\text {runs }}$ and the thermal fluctuation $\sigma_{\text {thermal }}$.

\begin{tabular}{|c|c|c|c|}
\hline & $\left\langle n_{\text {Hbond }}\right\rangle$ & $\sigma_{\text {runs }}$ & $\sigma_{\text {thermal }}$ \\
\hline & \multicolumn{3}{|c|}{${ }^{*} \mathrm{CO}$} \\
\hline $\mathrm{Cu}(211)$ & 0.28 & 0.05 & 0.51 \\
\hline $\mathrm{Cu}(111)$ & 0.32 & 0.03 & 0.53 \\
\hline $\mathrm{Au}(111)$ & 0.11 & 0.07 & 0.28 \\
\hline \multirow{2}{*}{$\operatorname{Pt}(111)$} & 0.44 & 0.05 & 0.62 \\
\hline & \multicolumn{3}{|c|}{${ }^{*} \mathrm{CHO}$} \\
\hline \multirow[t]{2}{*}{$\mathrm{Cu}(211)$} & 1.16 & 0.13 & 0.82 \\
\hline & \multicolumn{3}{|c|}{${ }^{*} \mathrm{COH}$} \\
\hline \multirow[t]{2}{*}{$\mathrm{Cu}(211)$} & 1.77 & 0.27 & 0.73 \\
\hline & \multicolumn{3}{|c|}{ *OCCHO } \\
\hline \multirow[t]{2}{*}{$\mathrm{Cu}(211)$} & 2.98 & 0.67 & 1.06 \\
\hline & \multicolumn{3}{|c|}{${ }^{*} \mathrm{OH}$} \\
\hline $\mathrm{Cu}(211)$ & 2.04 & 0.21 & 0.79 \\
\hline $\mathrm{Cu}(111)$ & 2.39 & 0.10 & 0.90 \\
\hline $\mathrm{Au}(111)$ & 2.96 & 0.02 & 0.69 \\
\hline \multirow[t]{2}{*}{$\operatorname{Pt}(111)$} & 2.76 & 0.07 & 0.62 \\
\hline & \multicolumn{3}{|c|}{${ }^{*} \mathrm{OOH}$} \\
\hline $\mathrm{Au}(111)$ & 3.42 & 0.08 & 0.85 \\
\hline $\operatorname{Pt}(111)$ & 2.91 & 0.37 & 0.82 \\
\hline
\end{tabular}

water adsorption on the otherwise very hydrophobic $\mathrm{Au}(111)$ surface. It should be noted, however, that the changes in water adsorption do not directly correlate with the changes in water density if an adsorbate is present. With few exceptions the adsorbate almost always decreases water adsorption. 


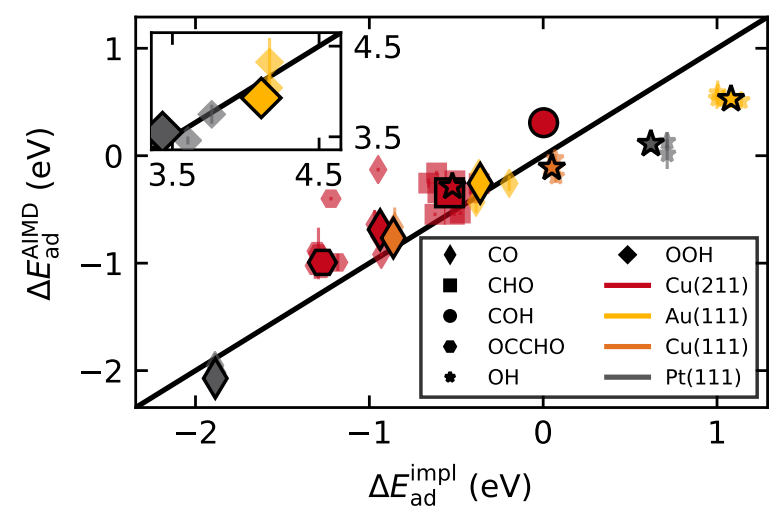

FIG. 7. Parity plot of adsorption energies obtained from AIMD simulations ( $\left.\Delta E_{\mathrm{ad}}^{\mathrm{AIMD}}\right)$ and geometry optimizations including implicit solvent $\left(\Delta E_{\text {ad }}^{\text {impl }}\right)$. The smaller symbols represent single-site energies determined from the two methods and the larger, framed symbols compare the average adsorption energy from AIMD vs. site-averaged energies from implicit solvation. The inset shows the data for the ${ }^{*} \mathrm{OOH}$ adsorbates.

\section{B. Stabilization through solvation}

We compare the adsorption energies (following eq. 1) for all adsorbates on the various surfaces as obtained from AIMD simulations and through implicit solvation. In general, this comparison is complicated due to the diffusion of the adsorbates onto different sites during the AIMD trajectories (c.f. Sec. II B). We probed the adsorption energies at all sites visited during the AIMD simulations via geometry optimizations in presence of continuum solvation. From the total share of sites observed during the AIMD simulations, we additionally evaluate a site-averaged adsorption energy from the geometry optimizations on the single sites, replicating the AIMD statistics. Fig. 7 shows the parity plot comparing the adsorption energies derived from AIMD and implicit solvation. The smaller symbols represent singlesite energies from the two methods and the larger, framed symbols compare the averaged adsorption energy from AIMD vs. site-averaged energies from implicit solvation. The single site energies generally cluster around the averaged energies, but a few outliers are present, which likely arise from insufficient statistics during the AIMD. As further indicated in Fig. 8, the site-averaged energies obtained from the geometry optimizations nearly coincide with the energy of the most stable sites. This confirms the validity of the usual Boltzmann weighted assumption to consider the most stable site only ${ }^{51}$ for static calculations. 
The deviation between adsorption energies obtained through implicit solvent and from AIMD appears to vary strongly across adsorbates and surfaces, as depicted in Fig. 8. On the one hand, implicit solvation overestimates the binding strength of all adsorbates on $\mathrm{Cu}(211)$ by a near constant $0.2-0.3 \mathrm{eV}$ and of ${ }^{*} \mathrm{OOH}$ on $\mathrm{Pt}(111)$ by $0.15 \mathrm{eV}$. On the other hand, for (111) terraces, implicit solvation underestimates the ${ }^{*} \mathrm{OH}$ binding strength by 0.3 to $0.6 \mathrm{eV}$ and the ${ }^{*} \mathrm{OOH}$ binding strength on $\mathrm{Au}(111)$ by $0.2 \mathrm{eV}$, while capturing the ${ }^{*} \mathrm{CO}$ binding strength to within $\pm 0.1 \mathrm{eV}$ of AIMD. To estimate the influence of the different solvent methods we compute the solvation energy $\Delta E_{\text {solv }}$ via

$$
\Delta E_{\text {solv }}=\Delta E_{\text {ad }}^{\text {solv }}-\Delta E_{\text {ad }}^{\mathrm{vac}}
$$

where $\Delta E_{\text {ad }}^{\text {solv }}$ is the adsorption energy including solvent and $\Delta E_{\text {ad }}^{\text {vac }}$ the adsorption energy without solvent. As shown in Fig. 8, the effect of the continuum solvent method always yields a minor stabilization proportional to the size of the adsorbate. In contrast to this, the solvation in the AIMD simulations yield both destabilization for i.e. most adsorbates on the $\mathrm{Cu}(211)$ facet as well as strong stabilization in the case of * $\mathrm{OH}$ on the (111) terraces.

The difference between adsorption energies determined using AIMD vs. continuum solvent is essentially accounted for by the differences between the respective solvation energies, within a certainty of $0.1 \mathrm{eV}$, as shown in Fig. 9. This correlation shows that the difference in the evaluated adsorption energies stems from the treatment of solvation and not the temperature difference, i.e. $0 \mathrm{~K}$ vs. $300 \mathrm{~K}$. For the most part, the minor deviation between solvation and adsorption energy differences can be explained by dynamic effects. For example, ${ }^{*} \mathrm{CO}$ on $\mathrm{Au}(111)$ is unstable on the surface. Through the sampling of surface sites during the AIMD we expect stabilization through mobility to manifest itself in different magnitude with and without solvent. To a smaller degree, a slight deviation in the adsorption energies is present because of the symmetric vs. non-symmetric slab models used in geometry optimization and AIMD, respectively (c.f. SI Sec. VIIIb). This effect does not affect the solvation energies. This difference in turn is ultimately seen in an apparent difference in solvation energy $\Delta E_{\mathrm{solv}}^{\mathrm{AIMD}}$ as compared to the difference in adsorption energy $\Delta E_{\mathrm{ad}}^{\mathrm{AIMD}}$.

We estimate the overall performance of the continuum solvation against AIMD for our benchmark set via the mean average error (MAE) in adsorption and solvation energies. We find the MAE for the adsorption energies to yield $0.24 \mathrm{eV}$ which is overall well reflected in the MAE for the solvation energy of $0.22 \mathrm{eV}$. In comparison, the MAE of the adsorption 

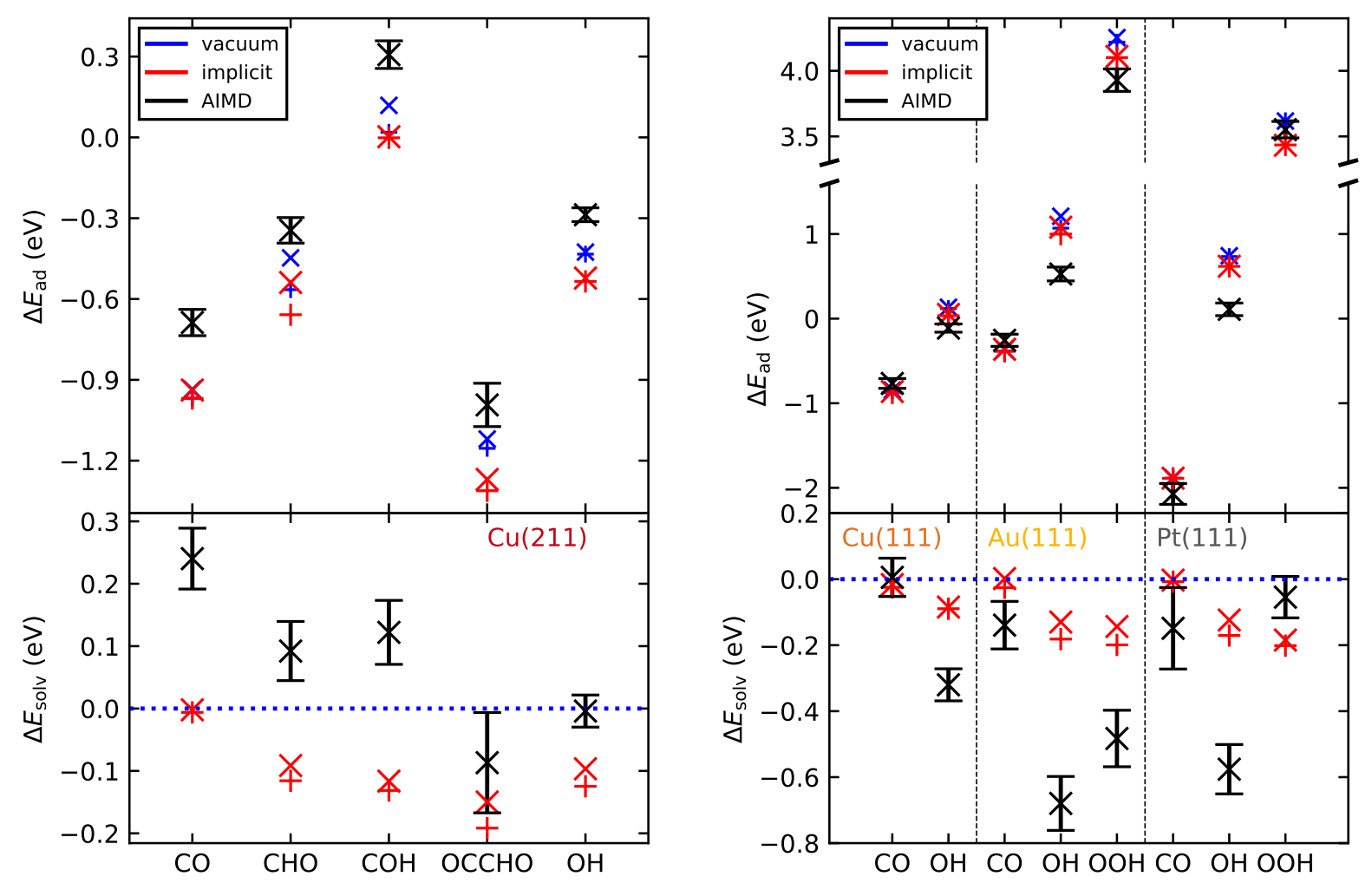

FIG. 8. Adsorption $\Delta E_{\text {ad }}$ (top) and solvation energies $\Delta E_{\text {solv }}$ (bottom) for the adsorbates sampled on $\mathrm{Cu}(211)$ (left) and $\mathrm{Cu}(111), \mathrm{Au}(111)$, and $\mathrm{Pt}(111)$ (right). Black symbols with error bars present the AIMD data, red and blue symbols data obtained through geometry optimizations with and without implicit solvent. $\times$ symbols represent time or site averaged data and + symbols the most stable configuration obtained from geometry optimizations. Data are tabulated in SI Sec. III.

energies in static calculations without solvent in vacuum $\left(\Delta E_{\mathrm{ad}}^{\mathrm{vac}}\right)$ is $0.25 \mathrm{eV}$. The similarity of the MAE of the vacuum and implicit solvent simulations suggest that implicit solvent models do not improve the accuracy of adsorption energies over vacuum simulations. This is also shown in Fig. 8, where the binding energies do not differ significantly when neglecting continuum solvent. However, this effect may be specific for strongly solvated adsorbates considered in the present work. It should also be noted, that we find the continuum solvent to predict geometries of solvated adsorbates more accurately, i.e. in the case of the dihedral angle of ${ }^{*} \mathrm{OCCHO}$ on $\mathrm{Cu}(211)$. 


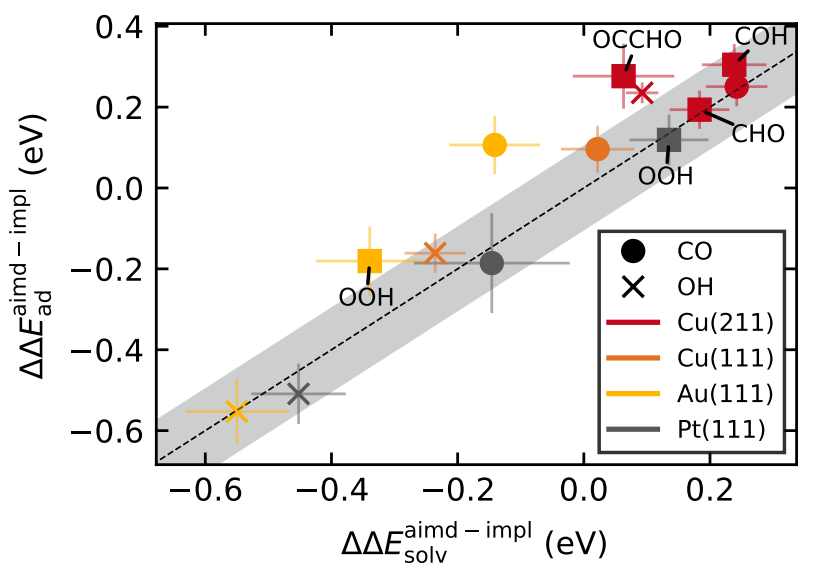

FIG. 9. The difference between adsorption energies determined using AIMD vs. continuum solvent, $\Delta \Delta E_{\text {ad }}^{\text {aimd-impl }}$, vs. the differences between the respective solvation energies, $\Delta \Delta E_{\text {solv }}^{\text {aimdimpl }}$. The shaded region indicates data points showing the same deviation in adsorption and solvation energy within $0.1 \mathrm{eV}$.

\section{Influence of thermalized explicit water}

We hypothesize that the main contributions to the AIMD solvation energies arise from hydrogen bonding and competitive water adsorption (c.f. Tab. II and Fig. 6). These phenomena also present the most fundamental differences to the continuum solvent model. The lack of these contributions thus rationalizes the large deviation in the solvation energies and limits the advantage in the application of continuum solvation over basic solid/vacuum simulations.

As demonstrated in Fig. 10, the AIMD solvation energies suggest a strong correlation with hydrogen bonding for ${ }^{*} \mathrm{OH}$ on all surfaces and ${ }^{*} \mathrm{COH}$ on $\mathrm{Cu}(211)$. These adsorbates present a subset which is distinguished by an $\mathrm{H}$ donating - $\mathrm{OH}$ group and small adsorbate size. The unambiguous relationship between hydrogen bonding and solvation energy for the $\mathrm{H}$ donating groups follows a linear function $\left(f=1.44 \mathrm{eV}-\left(0.72 \cdot\left\langle n_{\text {Hbond }}\right\rangle\right) \mathrm{eV}\right)$. We also find a correlation between competitive water adsorption and the solvation energy for ${ }^{*} \mathrm{CO}$ on all surfaces and ${ }^{*} \mathrm{CHO}$ on $\mathrm{Cu}(211)$. This correlation is captured in the difference in the total water adsorption between the clean surface and in the presence of an adsorbate, i.e. the replacement of adsorbing water (giving a linear relationship as $f=-0.15 \mathrm{eV}-$ $\left.\left(0.68 \cdot \Delta\left\langle n_{\text {ads }}^{\mathrm{H}_{2} \mathrm{O}}\right\rangle\right) \mathrm{eV}\right)$. This relationship reveals a basic stabilization when no competitive 

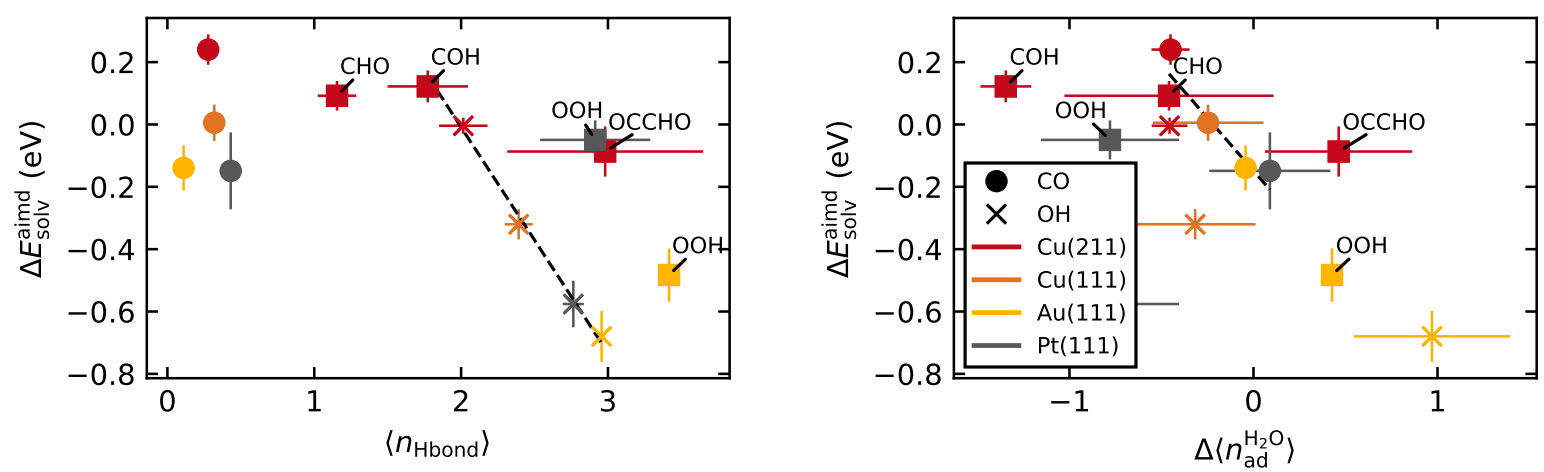

FIG. 10. AIMD solvation energy $\Delta E_{\text {solv }}^{\text {aimd }}$ against the average number of hydrogen bonds $\left\langle n_{\text {Hbond }}\right\rangle$ (left) and against the difference in average number of adsorbed water molecules in presence of an adsorbate compared to the pure metal/water interface $\Delta\left\langle n_{\text {ads }}^{\mathrm{H}_{2} \mathrm{O}}\right\rangle$ (right). Apparent correlations derived from a weighted fit (see text) are indicated by the dark dashed lines. Data are tabulated in Tab. I and in the SI Sec. III and IV.

water adsorption is present (i.e. $\approx-0.15 \mathrm{eV}$ for ${ }^{*} \mathrm{CO}$ on $\operatorname{Pt}(111)$ and $\mathrm{Au}(111)$ ). Since we find no apparent correlation with hydrogen bonding for these cases, this stabilization may be linked to the cavity formation in the water. The competitive water adsorption also correlates for some of the ${ }^{*} \mathrm{OH}$ adsorbates. Since hydrogen bonding and competitive water adsorption are related properties defined by the nature of the solid/liquid interface, this apparent correlation is not surprising. The observed correlations of the small adsorbates can also serve as a correction to continuum solvation energies. As shown in Fig. S4 in the SI, nearly the same relationships apply to the deviation in solvation energies. Less clearly correlated are ${ }^{*} \mathrm{OOH}$ on $\mathrm{Pt}(111), \mathrm{Au}(111)$ and ${ }^{*} \mathrm{OCCHO}$ on $\mathrm{Cu}(211)$. The ${ }^{*} \mathrm{OOH}$ adsorbates follow a similar, parallel shifted trend for hydrogen bonding as $* \mathrm{OH}$ and ${ }^{*} \mathrm{COH}$ (c.f. SI Sec. Vb). Additionally, *OCCHO appears to align in that latter relation. Due to the increased size of these adsorbates and their multiple -O and -OH groups viable for hydrogen bonding, ${ }^{*} \mathrm{OOH}$ and ${ }^{*} \mathrm{OCCHO}$ may follow a common hydrogen bonding trend. However, our limited number of data points can only give tentative insight. Additionally, the statistics for $* \mathrm{OCCHO}$ on $\mathrm{Cu}(211)$ and $* \mathrm{OOH}$ on $\mathrm{Pt}(111)$ show strong variation in (at least) the hydrogen bonding. The corresponding trajectories exhibit many configurational changes, chemical reactions or necessitated some discard of data (c.f. Sec. III A and SI Sec. VIIIa). 
The systematic correlation of the solvation energies with hydrogen bonding $\left\langle n_{\text {Hbond }}\right\rangle$ and competitive water adsorption $\Delta\left\langle n_{\text {ads }}^{\mathrm{H}_{2} \mathrm{O}}\right\rangle$ suggest a phenomenological model to determine adsorbate solvation energies a priori. Such a scheme would be highly desirable to circumvent costly AIMD simulations for each adsorbate-surface combination. In that sense, easy-todetermine descriptors would be necessary from which $\left\langle n_{\text {Hbond }}\right\rangle$ and $\Delta\left\langle n_{\text {ads }}^{\mathrm{H}_{2} \mathrm{O}}\right\rangle$ may be predicted. As further described in the SI Sec. Vb, we survey a number of descriptors. In these, we do not find unambiguous correlations sufficient for a general a priori model. Although correlations could be non-trivial, their absence may be circumstantial to a lack of data points. Notwithstanding, we find scaling-relation-like ${ }^{55}$ correlations between hydrogen bonding of ${ }^{*} \mathrm{OH}$ and its adsorption energy at the metal/vacuum interface without presence of any water molecule $\left(\Delta E_{\mathrm{ad}}^{\mathrm{vac},{ }^{*} \mathrm{OH}}\right)$ at the limit of zero coverage. From this correlation we can formulate a model to predict solvation energies specifically for $* \mathrm{OH}$ on metal surfaces. A function for the solvation energy of $* \mathrm{OH}\left(\Delta E_{\mathrm{solv}}^{* \mathrm{OH}}\right)$ follows as

$$
\Delta E_{\mathrm{solv}}^{* \mathrm{OH}}=-0.22 \mathrm{eV}-0.44 \cdot \Delta E_{\mathrm{ad}}^{\mathrm{vac},{ }^{*} \mathrm{OH}} .
$$

Eq. 4 suggests a stronger solvation stabilization of ${ }^{*} \mathrm{OH}$ in the case of weaker binding to the metal. Further, we are also able to quantitatively predict the total water adsorption from $\mathrm{H}_{2} \mathrm{O}$ binding energies. The existence of these trends indicates fundamental atomistic relationships with the metal/water interface and thus a possibility for general a priori models.

\section{Comparison to other explicit solvation approaches}

We compare the solvation energies from our AIMD simulations to literature reports, as summarized in Tab. III. We restrict the comparison to results obtained by inclusion of explicit water molecules and omit implicit solvation methods.

Since available AIMD data points are scarce in literature, we also include solvation energies determined from classical molecular dynamics and DFT single point energies ${ }^{56,57}$. Regardless, comparable data to our test set is still limited to the electrochemical model system $\mathrm{Pt}(111)$. We find good agreement for ${ }^{*} \mathrm{CO}^{56}$ but only limited agreement for $* \mathrm{OH}^{44}$ on $\mathrm{Pt}(111)$. For the latter, we found a deviation of $-0.26 \mathrm{eV}$, where it should be noted that the considered reactions differ, i.e. $\mathrm{H}_{2} \mathrm{O}(\mathrm{g}) \rightarrow{ }^{*} \mathrm{OH}+\frac{1}{2} \mathrm{H}_{2}(\mathrm{~g})$ (our study) vs. $\mathrm{H}_{2} \mathrm{O}^{*} \rightarrow{ }^{*} \mathrm{OH}$ $+\frac{1}{2} \mathrm{H}_{2}(\mathrm{~g})$. 
TABLE III. Solvation energies in eV for different adsorbates on $\mathrm{Cu}(211), \mathrm{Cu}(111), \mathrm{Au}(111)$, and Pt(111) from this work (left column) and adapted from literature based on different methods. *indicate an approximation to AIMD via classical molecular dynamics and single point DFT energies.

\begin{tabular}{|c|c|c|c|}
\hline & this work & AIMD & static bilayer \\
\hline & \multicolumn{3}{|c|}{$\mathrm{Cu}(211)$} \\
\hline$* \mathrm{CO}$ & $0.24 \pm 0.05$ & - & $-0.2^{58,59} /-0.1^{16}$ \\
\hline${ }^{*} \mathrm{CHO}$ & $0.09 \pm 0.05$ & - & $-0.3^{58,59} /-0.15^{16}$ \\
\hline$* \mathrm{COH}$ & $0.12 \pm 0.05$ & - & $-0.2^{16}$ \\
\hline *OCCHO & $-0.09 \pm 0.08$ & - & $+0.05^{16}$ \\
\hline \multirow[t]{2}{*}{$* \mathrm{OH}$} & $0.0 \pm 0.03$ & - & $-0.13^{59}$ \\
\hline & \multicolumn{3}{|c|}{$\mathrm{Cu}(111)$} \\
\hline$* \mathrm{CO}$ & $0.01 \pm 0.06$ & - & $-0.1^{60}$ \\
\hline \multirow[t]{2}{*}{$* \mathrm{OH}$} & $-0.32 \pm 0.05$ & - & - \\
\hline & \multicolumn{3}{|c|}{$\mathrm{Au}(111)$} \\
\hline$* \mathrm{CO}$ & $-0.14 \pm 0.07$ & - & - \\
\hline$* \mathrm{OH}$ & $-0.68 \pm 0.08$ & - & - \\
\hline \multirow[t]{2}{*}{$* \mathrm{OOH}$} & $-0.48 \pm 0.09$ & - & - \\
\hline & \multicolumn{3}{|c|}{$\operatorname{Pt}(111)$} \\
\hline$* \mathrm{CO}$ & $-0.15 \pm 0.12$ & $01 \pm 0.09^{56 *}$ & - \\
\hline$* \mathrm{OH}$ & $-0.58 \pm 0.07$ & $-0.32^{44}$ & $-0.4^{61} /-0.6^{62}$ \\
\hline$* \mathrm{OOH}$ & $-0.05 \pm 0.06$ & - & $-0.5^{7} /-0.7^{62}$ \\
\hline
\end{tabular}

In contrast to AIMD simulations, solvation energies from explicit ice-like water bilayer models have been reported significantly more frequently. These solvation energies are usually consistently obtained using only one ice-like bilayer ${ }^{63}$. Where available, we find the deviation between reported solvation energies to vary within $0.1-0.2 \mathrm{eV}$ which may very well be due to uncertainties in sampling of the water structure. The solvation energies determined at terrace sites compare relatively well (within the uncertainties) to our AIMD results in most cases. Only the stabilization of ${ }^{*} \mathrm{CO}$ on $\mathrm{Cu}(111)$ is slightly and ${ }^{*} \mathrm{OOH}$ on $\mathrm{Pt}(111)$ is strongly overestimated in the static picture. Considerably less agreement is found for solva- 
tion energies on the $\mathrm{Cu}(211)$ step. All static water layer energies indicate comparably strong stabilization while the AIMD results indicate destabilization (with the exception of the more complicated $\left.{ }^{*} \mathrm{OCCHO}\right)$. This trend likely arises from competitive water adsorption, which is very prominent on the step sites and is seemingly not captured in a static calculation. On another note, there is a difference in solvation energies of ${ }^{*} \mathrm{OH}$ on the $\mathrm{Cu}(211)$ and $\mathrm{Pt}(111)$ surface in the case of static water calculations. Such a variation of the same adsorbate on different metal surfaces is similar to our AIMD results. It follows that the solvation energies for a given adsorbate on different surfaces cannot necessarily be assumed to be the same.

\section{SUMMARY AND CONCLUSION}

We benchmarked solvation energies of adsorbates at the metal/water interface as obtained from continuum solvation methods against AIMD simulations. We considered ${ }^{*} \mathrm{CO},{ }^{*} \mathrm{OH}$ on $\mathrm{Cu}(111), \mathrm{Au}(111)$, and $\mathrm{Pt}(111),{ }^{*} \mathrm{OOH}$ on $\mathrm{Au}(111)$ and $\mathrm{Pt}(111)$ as well as ${ }^{*} \mathrm{CO},{ }^{*} \mathrm{CHO}$, ${ }^{*} \mathrm{COH},{ }^{*} \mathrm{OCCHO}$, and ${ }^{*} \mathrm{OH}$ on $\mathrm{Cu}(211)$. These highly different adsorbates, the simple terrace surfaces, and the complex $\mathrm{Cu}(211)$ stepped surface constitute a representative test-set for solvation effects on metal surfaces. We find the deviation between AIMD and continuum solvation to be substantial, especially for strongly solvated adsorbates such as * $\mathrm{OH}$ and *OOH. In that, based on the MAE in relation to our AIMD results, we find no apparent advantage in applying continuum solvation in comparison to a basic solid/vacuum calculation to determine adsorption energies at the metal/water interface. We want to remark, that this conclusion does not include implicit solvent models which account for directional interactions (e.g. CANDLE ${ }^{12}$ ). Moreover, ongoing developments in continuum solvation ${ }^{13,64-66}$ could reconcile the present shortcomings. Further, we find that solvation energies for individual adsorbates vary significantly between metals and even facets, which puts to question the common assumption that solvation energies of given adsorbates do not change with metal surface identity.

We identify hydrogen bonding and competitive water adsorption as the main contributors to the solvation energy captured in AIMD simulations. This insight is supported by quantitative relationships between these phenomena and solvation energies. Specifically, we find that small adsorbates with hydrogen donating $-\mathrm{OH}$ groups $\left({ }^{*} \mathrm{OH},{ }^{*} \mathrm{COH}\right)$ are stabilized by hydrogen bonding and weakly interacting adsorbates $\left({ }^{*} \mathrm{CO},{ }^{*} \mathrm{CHO}\right)$ are destabilized by 
competitive water adsorption. The larger adsorbates $* \mathrm{OOH}$ and $* \mathrm{OCCHO}$ appear to follow a separate correlation which we, however, cannot unambiguously define. The discovered relationships rationalize trends of solvation among different adsorbates, facets, and metals. Also, these relationships may be a basis for a general systematic a priori thermodynamic treatment to account for solvation effects. However, the immediate, general prediction of the underlying descriptors - average hydrogen bonding and competitive water adsorption - via reliable surface/adsorbate properties appears non-trivial, and is the subject of future work.

\section{ACKNOWLEDGMENTS}

Financial support was provided by V-Sustain: The VILLUM Centre for the Science of Sustainable Fuels and Chemicals (\#9455) from VILLUM FONDEN. J.A.G. gratefully acknowledges support from the Joint Center for Artificial Photosynthesis, a DOE Energy Innovation Hub, supported through the Office of Science of the U.S. Department of Energy under Award Number DE-SC0004993. T.L. acknowledges support from the United States Department of Energy through the Computational Sciences Graduate Fellowship (DOE CSGF) under grant number DE-FG02-97ER25308. H.H.H. thanks Vanessa Jane Bukas for valuable discussions.

\section{REFERENCES}

${ }^{1}$ O. M. Magnussen and A. Groß, J. Am. Chem. Soc. 141, 4777 (2019).

${ }^{2}$ J. Nørskov, J. Rossmeisl, A. Logadottir, L. Lindqvist, J. Kitchin, T. Bligaard, and H. Jónsson, J. Phys. Chem. B 108, 17886 (2004).

${ }^{3}$ S. Schnur and A. Groß, Catal. Today 165, 129 (2011).

${ }^{4}$ O. Andreussi, I. Dabo, and N. Marzari, J. Chem. Phys. 136 (2012), 10.1063/1.3676407.

${ }^{5}$ K. Mathew and R. G. Hennig, arXiv preprint (2016), arXiv:1601.03346.

${ }^{6}$ J. Rossmeisl, E. Skúlason, M. E. Björketun, V. Tripkovic, and J. K. Nørskov, Chem. Phys. Lett. 466, 68 (2008).

${ }^{7}$ V. Tripković, E. Skúlason, S. Siahrostami, J. K. Nørskov, and J. Rossmeisl, Electrochimica Acta 55, 7975 (2010). 
${ }^{8}$ C. Taylor, R. G. Kelly, and M. Neurock, J. Electrochem. Soc. 153, E207 (2006).

${ }^{9}$ S. Schnur and A. Groß, New J. Phys. 11, 125003 (2009).

${ }^{10}$ J.-B. Le and J. Cheng, Curr. Opin. Electrochem. (2019), 10.1016/j.coelec.2019.11.008.

${ }^{11}$ D. Chandler and H. C. Andersen, J. Chem. Phys. 57, 1930 (1972).

${ }^{12}$ R. Sundararaman and W. A. Goddard III, J. Chem. Phys. 142, 064107 (2015).

${ }^{13}$ R. Sundararaman and K. Schwarz, The Journal of chemical physics 146, 084111 (2017).

${ }^{14}$ F. Nattino, M. Truscott, N. Marzari, and O. Andreussi, J. Chem. Phys. 150, 041722 (2019).

${ }^{15}$ N. G. Hörmann, O. Andreussi, and N. Marzari, J. Chem. Phys. 150, 041730 (2019).

${ }^{16}$ T. Ludwig, J. A. Gauthier, K. S. Brown, S. Ringe, J. K. Nørskov, and K. Chan, J. Phys. Chem. C 123, 5999 (2019).

${ }^{17}$ G. Kastlunger, P. Lindgren, and A. A. Peterson, J. Phys. Chem. C 122, 12771 (2018).

${ }^{18}$ J. A. Gauthier, C. F. Dickens, S. Ringe, and K. Chan, ChemPhysChem (2019), 10.1002/cphc.201900536.

${ }^{19}$ J. A. Gauthier, C. F. Dickens, H. H. Heenen, V. Sudarshan, S. Ringe, and K. Chan, J. Chem. Theory Comput. (2019), 10.1021/acs.jctc.9b00717.

${ }^{20}$ N. G. Hörmann, Z. Guo, F. Ambrosio, O. Andreussi, A. Pasquarello, and N. Marzari, Npj Comput. Mater. 5, 1 (2019).

${ }^{21}$ M. M. Melander, M. J. Kuisma, T. E. K. Christensen, and K. Honkala, J. Chem. Phys. 150, 041706 (2019).

${ }^{22}$ S. Surendralal, M. Todorova, M. W. Finnis, and J. Neugebauer, Phys. Rev. Lett. 120, 246801 (2018).

${ }^{23}$ R. Khatib, A. Kumar, S. Sanvito, M. Sulpizi, and C. S. Cucinotta, arXiv preprint arXiv:1905.11850 (2019).

${ }^{24}$ H. H. Kristoffersen and J. H. Chang, in Computational Photocatalysis: Modeling of Photophysics and Photochemistry at Interfaces (ACS Publications, 2019) pp. 225-238.

${ }^{25}$ G. Kresse and J. Furthmüller, Phys. Rev. B: Condens. Matter Mater. Phys. 54, 11169 (1996).

${ }^{26}$ G. Kresse and J. Furthmüller, Comput. Mater. Sci. 6, 15 (1996).

${ }^{27}$ P. Blöchl, Phys. Rev. B: Condens. Matter Mater. Phys. 50, 17953 (1994).

${ }^{28}$ B. Hammer, L. B. Hansen, and J. K. Nørskov, Phys. Rev. B 59, 7413 (1999).

${ }^{29}$ S. Grimme, J. Antony, S. Ehrlich, and H. Krieg, J. Chem. Phys. 132, 154104 (2010). 
${ }^{30}$ S. Grimme, Wiley Interdiscip. Rev.: Comput. Mol. Sci. 1, 211 (2011).

${ }^{31}$ K. Tonigold and A. Groß, J. Comput. Chem. 33, 695 (2012).

${ }^{32}$ S. Sakong, K. Forster-Tonigold, and G. Axel, J. Chem. Phys. 144, 194701 (2016).

${ }^{33}$ S. Sakong and G. Axel, J. Chem. Phys. 149, 084705 (2018).

${ }^{34}$ S. Nosé, J. Chem. Phys. 81, 511 (1984).

${ }^{35}$ S. Nosé, Prog. Theor. Phys. Suppl. 103, 1 (1991).

${ }^{36}$ D. Bylander and L. Kleinman, Phys. Rev. B: Condens. Matter Mater. Phys. 46, 13756 (1992).

${ }^{37}$ H. Monkhorst and J. Pack, Phys. Rev. B: Condens. Matter. 13, 5188 (1976).

${ }^{38}$ L. Bengtsson, Phys. Rev. B 59, 12301 (1999).

${ }^{39}$ A. H. Larsen, J. J. Mortensen, J. Blomqvist, I. E. Castelli, R. Christensen, M. Dułak, J. Friis, M. N. Groves, B. Hammer, C. Hargus, E. D. Hermes, P. C. Jennings, P. B. Jensen, J. Kermode, J. R. Kitchin, E. L. Kolsbjerg, J. Kubal, K. Kaasbjerg, S. Lysgaard, J. B. Maronsson, T. Maxson, T. Olsen, L. Pastewka, A. Peterson, C. Rostgaard, J. Schiøtz, O. Schütt, M. Strange, K. S. Thygesen, T. Vegge, L. Vilhelmsen, M. Walter, Z. Zeng, and K. W. Jacobsen, J. Phys. Condens. Matter 29, 273002 (2017).

${ }^{40}$ D. Gunceler, K. Letchworth-Weaver, R. Sundararaman, K. A. Schwarz, and T. Arias, Modelling Simul. Mater. Sci. Eng. 21, 074005 (2013).

${ }^{41}$ P. Giannozzi, S. Baroni, N. Bonini, M. Calandra, R. Car, C. Cavazzoni, D. Ceresoli, G. L. Chiarotti, M. Cococcioni, I. Dabo, A. D. Corso, S. de Gironcoli, S. Fabris, G. Fratesi, R. Gebauer, U. Gerstmann, C. Gougoussis, A. Kokalj, M. Lazzeri, L. Martin-Samos, N. Marzari, F. Mauri, R. Mazzarello, S. Paolini, A. Pasquarello, L. Paulatto, C. Sbraccia, S. Scandolo, G. Sclauzero, A. P. Seitsonen, A. Smogunov, P. Umari, and R. M. Wentzcovitch, J. Phys. Condens. Matter 21, 395502 (2009).

${ }^{42}$ S. K. Natarajan and J. Behler, Phys. Chem. Chem. Phys. 18, 28704 (2016).

${ }^{43}$ H. H. Kristoffersen, J.-E. Shea, and H. Metiu, J. Phys. Chem. Lett. 6, 2277 (2015).

${ }^{44}$ H. H. Kristoffersen, T. Vegge, and H. A. Hansen, Chem. Sci. 9, 6912 (2018).

${ }^{45}$ E. Gallicchio, M. Kubo, and R. M. Levy, J. Phys. Chem. B 104, 6271 (2000).

${ }^{46}$ D. Frenkel and B. Smit, in Understanding Molecular Simulation: From Algorithms to Applications (Akademic Press, 1996).

${ }^{47}$ H. Flyvbjerg and H. Petersen, J. Chem. Phys. 91, 461 (1989).

${ }^{48}$ J. R. Boes, O. Mamun, K. Winther, and T. Bligaard, J. Phys. Chem. A 123, 2281 (2019). 
${ }^{49}$ A. Soper, F. Bruni, and M. Ricci, J. Chem. Phys. 106, 247 (1997).

${ }^{50}$ P. Gono, F. Ambrosio, and A. Pasquarello, J. Phys. Chem. C 123, 18467 (2019).

${ }^{51}$ J. K. Nørskov, F. Studt, F. Abild-Pedersen, and T. Bligaard, Fundamental Concepts in Heterogeneous Catalysis (John Wiley \& Sons, Inc., 2014).

${ }^{52}$ R. Mills, J. Phys. Chem. 77, 685 (1973).

${ }^{53}$ I.-C. Lin, A. P. Seitsonen, I. Tavernelli, and U. Rothlisberger, J. Chem. Theory Comput. 8, $3902(2012)$.

${ }^{54}$ A. Groß, F. Gossenberger, X. Lin, M. Naderian, S. Sakong, and T. Roman, J. Electrochem. Soc. 161, E3015 (2014).

${ }^{55}$ F. Abild-Pedersen, J. Greeley, F. Studt, J. Rossmeisl, T. Munter, P. G. Moses, E. Skulason, T. Bligaard, and J. K. Nørskov, Phys. Rev. Lett. 99, 016105 (2007).

${ }^{56}$ C. J. Bodenschatz, S. Sarupria, and R. B. Getman, J. Phys. Chem. C 119, 13642 (2015).

${ }^{57}$ V. J. Bukas, H. W. Kim, R. Sengpiel, K. Knudsen, J. Voss, B. D. McCloskey, and A. C. Luntz, ACS Catal. 8, 11940 (2018).

${ }^{58}$ X. Liu, J. Xiao, H. Peng, X. Hong, K. Chan, and J. K. Nørskov, Nat. Commun. 8 (2017), $10.1038 /$ ncomms15438.

${ }^{59}$ X. Liu, P. Schlexer, J. Xiao, Y. Ji, L. Wang, R. B. Sandberg, M. Tang, K. S. Brown, H. Peng, S. Ringe, C. Hahn, T. F. Jaramillo, J. K. Nørskov, and K. Chan, Nat. Commun. 10 (2019), 10.1038/s41467-018-07970-9.

${ }^{60}$ A. A. Peterson, F. Abild-Pedersen, F. Studt, J. Rossmeisl, and J. K. Nørskov, Energy Environ. Sci. 3, 1311 (2010).

${ }^{61}$ G. Karlberg, Phys. Rev. B 74, 153414 (2006).

${ }^{62}$ S. Liu, M. G. White, and P. Liu, J. Phys. Chem. C 120, 15288 (2016).

${ }^{63}$ J. A. Gauthier, C. F. Dickens, L. D. Chen, A. D. Doyle, and J. K. Nørskov, J. Phys. Chem. C 21, 11455 (2017).

${ }^{64}$ S. Ringe, H. Oberhofer, and K. Reuter, J. Chem. Phys. 146, 134103 (2017).

${ }^{65}$ C. Hille, S. Ringe, M. Deimel, C. Kunkel, W. E. Acree, K. Reuter, and H. Oberhofer, J. Chem. Phys. 150, 041710 (2019).

${ }^{66}$ O. Andreussi, N. G. Hörmann, F. Nattino, G. Fisicaro, S. Goedecker, and N. Marzari, J. Chem. Theory Comput. 15, 1996 (2019). 